\title{
Elaboración del Plan de Desarrollo y Ordenamiento Territorial Caso Gobierno autónomo descentralizado de Calpi 2011 (Competencia del desarrollo parroquial)
}

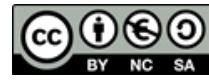

Preparation of the Development Plan and Territorial Ordering Case of the Decentralized Autonomous Government of Calpi 2011 (Parish development competence)

Eduardo Xavier Centeno Parra. ${ }^{1}$, Giovanny Javier Alarcón Parra. ${ }^{2}$, Jose Gabriel Pilaguano Mendoza. ${ }^{3}$ \& Charles Paul Viscarra Armijos. ${ }^{4}$

\begin{abstract}
.
DOI: https://doi.org/10.33262/concienciadigital.v4i2.2.1735

A proposal for the development plan and territorial ordering of the decentralized autonomous government of the parish of Santiago de Calpi focused on the competence of parish development was elaborated, According to the MDUV (2018) the main tool for planning development and territorial ordering at decentralized levels they are the PDOT. The PDOT is a management instrument to optimally channel economic resources allowing a correct investment that the parish, together with the autonomous decentralized cantonal and provincial governments, began the investigation with the participation of the presidents of the communities and neighborhoods where The problems of the parish were analyzed through consultation tables, surveys, interviews, in conclusion based on the competence of the parish development, the following programs were determined, monitoring the fulfillment of PDOT goals, updating program of quality indicators of life
\end{abstract}

\footnotetext{
${ }^{1}$ Escuela Superior Politécnica de Chimborazo; Dirección de Evaluación y Aseguramiento de la Calidad; xavier.centeno@espoch.edu.ec; https://orcid.org/0000-0003-4311-7228

${ }^{2}$ Chimborazo; Escuela Superior Politécnica de Chimborazo carrera de empresas; galarcon@ espoch.edu.ec; https://orcid.org/0000-0001-8468-9316,

Chimborazo; Escuela Superior Politécnica de Chimborazo carrera de finanzas; gpilaguano@espoch.edu.ec https://orcid.org/0000-0002-7153-6879

4 Universidad Estatal de Bolívar - Carrera de Mercadotecnia, chviscarra@ueb.edu.ec, https://orcid.org/0000-0002-0247-4731
} 
in the rural sector, participatory development planning program, inter-institutional management program, program to strengthen agricultural-productive peasant organizations, and finally, the multi-year plan of minimum goals falls, investment matrix assigned by participatory budgeting in the instances $d$ and citizen participation and the multi-year budget execution plan.

Keywords: Management, planning, land use, budget.

\section{Resumen}

Se elaboró una propuesta del plan de desarrollo y ordenamiento territorial del gobierno autónomo descentralizado de la parroquia de Santiago de Calpi enfocado a la competencia del desarrollo parroquial, Según el MDUV (2018) la principal herramienta de planificación del desarrollo y ordenamiento territorial en los niveles descentralizados son los PDOT. El PDOT es un instrumento de gestión para canalizar de una manera óptima los recursos económicos permitiendo una correcta inversión que la parroquia en conjunto con los gobiernos autónomos descentralizados cantonal y provincial, se inició la investigación con la participación de los presidentes de las comunidades y barriales donde se analizaron las problemáticas de la parroquia a través de mesas de concertación, encuestas, entrevistas, en conclusión en base a las competencia del desarrollo parroquial se determinaron los siguientes programas, seguimiento del cumplimiento de metas del PDOT, programa de actualización de indicadores de calidad de vida en el sector rural, programa de planificación participativa del desarrollo, programa de gestión interinstitucional, programa de fortalecimiento de las organizaciones campesinas agro productivas, y por ultimo recaen al plan plurianual de acciones metas mínimas, matriz de inversión asignada por presupuestario participativa en las instancias de participación ciudadana y el plan plurianual de ejecución presupuestaria.

Palabras clave: Gestión, planificación, ordenamiento territorial, presupuesto.

\section{Introducción}

La Constitución de la República de Ecuador (2008) busca cumplir con el mandato de "planificar el desarrollo nacional, erradicar la pobreza, promover el desarrollo sustentable y la redistribución equitativa de los recursos y la riqueza, para acceder al buen vivir". (Asamblea Constitucional de Montecristi , 2008)

Planificar implica tener visión, entendida ésta como la representación mental o imagen objetivo del futuro deseable y posible. La visión articula la percepción de un futuro realista, creíble y atractivo para un individuo, un grupo una organización, una región un país con lo que ahora existe. Ella debe expresar las aspiraciones fundamentales de los diferentes actores. La visión debe ser alcanzable y debe definir la dirección del proceso de cambio. (Guzmán Saavedra , Castro Zea, Restrepo Quintero, \& Rojas Rojas, 2001) 
Para los Gobiernos Parroquiales es fundamental realizar su Planificación cada cinco años y actualizaciones cada dos años según Senplades, de tal manera es fundamental planificar con todos los actores de la Parroquia para de esta manera determinar las necesidades de la misma y brindar estrategias sustentables para poder mejorar la calidad de vida de los mismos

La acción de planificar es una tarea integral y multidisciplinar. La idea de planificar está ligada a la de prever, organizar, preparar y ordenar unas determinadas acciones que queremos realizar para conseguir algo más. Implica anticiparse al futuro para poder constituirlo de acuerdo a un modelo de realidad que se considera deseable (Alberich \& Sotomayor, 2014)

Los niveles de concreción de la planificación es el Plan.- Define a grandes rasgos las ideas que van a orientar y condicionar el resto de los niveles de la planificación; Programa.Concreta objetivos y los temas que se exponen en el Plan; Proyecto.- es el nivel operativo del proceso de planificación, hay que saber que los niveles pueden ser planificados a corto mediano y largo plazo de tiempo para su ejecución y seguimiento. (Serrano, 2016)

Los Planes de Desarrollo y Ordenamiento Territorial son herramientas de gestión que permite a los GAD'S Parroquiales fomentar el desarrollo de la sociedad y Población General, destinando los recursos económicos que recibe del gobierno central para la redistribución de los recursos.

En la actualidad el Gad Parroquial de Calpi tiene dificultades con la articulación de sus objetivos y cumplimientos de sus metas con respecto a la planificación y ordenamiento de su parroquia, pues no cuentan con un Plan de Ordenamiento Territorial en el cual puedan observar las dimensiones la zonificación territorial.

En los procesos de formulación y ejecución de las políticas públicas, se establecerán mecanismos de coordinación que garanticen la coherencia y complementariedad entre las intervenciones de los distintos niveles de gobierno. Para este efecto, los instrumentos de planificación de los GAD propiciarán la incorporación de las intervenciones que requieran la participación del nivel desconcentrado de la función ejecutiva; asimismo las entidades desconcentradas de la función ejecutiva, incorporarán en sus instrumentos de planificación las intervenciones que se ejecuten de manera concertada con los GAD. (STPE(2019))

En vista de la necesidad de mejorar los procesos el Estado, pone énfasis en una visión de satisfacer las necesidades básicas en la planificación y ordenamiento territorial con herramientas enfocadas en ámbitos políticos, normativos e institucionales de esta manera fortalecer la equitativa asignación territorial y la consolidación de la Planificación Participativa.

\section{Metodología}

La metodología fue proporcionada por la SENPLADES donde se aborda temáticas sobre las características generales de la parroquia, metodología de construcción del plan de 
desarrollo y ordenamiento territorial parroquial, diagnóstico por subsistemas de la parroquia Calpi, modelo de territorio actual, propuesta de desarrollo territorial, cabe mencionar que se utilizaran técnicas de investigación tales como mesas de socialización, encuestas, entrevistas que según Fernández (2002) indica que la investigación cualitativa trata de identificar la naturaleza profunda de las realidades, la relación y estructura dinámica, por otro lado la investigación cuantitativa trata de determinar la fuerza de las asociaciones o correlación entre variables, la generalización y objetivación de los resultados a través de una muestra para ser inferencia en una población.

En tal sentido la utilización de estos métodos nos permitió la elaboración del plan desarrollo al analizar instrumentos, técnicas que permitieron obtener los resultados para la recolección de información no obstante, la observación interna o participante de las comunidades se caracteriza por qué atribuye a investigación cualitativa, se observa y se registra todo lo que sucede en contacto con el contexto de observación, se consideran categorías de análisis emergentes. (Pascual, 2016)

\section{Resultados}

El PDOT es un instrumento técnico y normativo para la planificación territorial. Orienta las intervenciones de las instituciones públicas y privadas para generar el desarrollo local. En esencia, un PDOT es una propuesta para ordenar la gestión de un territorio, en armonía con los actores involucrados y de acuerdo a las vocaciones del territorio. Además, es un instrumento político, pues refleja la visión de desarrollo, estrategias, programas y proyectos que permiten alcanzar el plan de trabajo de la autoridad electa.( Plan de Desarrollo y Ordenamiento Territorial (PDOT, 2019))

Características Generales Del Territorio, se encuentra ubicada a 15 minutos del Cantón Riobamba pertenece a la provincia del Chimborazo posee 6469 habitantes su extensión 5353 ha su altitud 3200msnm. Clima Tropical mega térmico húmedo TMH

Características Ecológicas con respecto a la Fisiografía y Suelo se describe de dos clases de suelos unos suavemente inclinados, bien drenados y casi llanos de drenaje defectuoso, en los cuales el movimiento de las aguas es lento, debido a un subsuelo duro o plástico, que requiere de métodos sencillos de producción. El clima se ubica en la categoría de Tropical Mega térmico Húmedo TMH. La Orografía en la parroquia Calpi tiene una morfología sumamente accidental e irregular por la presencia de quebradas como El Duende, Suculahuan, Las Abras, San Francisco, Castong, Pucatuni, Cordovéz, Chacha, Huaycu y Nagujón; cuenta además con pajonales ubicados en las montañas. La Hidrología cuenta con un sistema hidrográfico importante constituido por los siguientes ríos, Rio Chimborazo, El Rio Chibunga Las Zonas de Vida tiene una variedad de cultivos en el sector: Maíz, choclo suave, Papa, Cebada, Arveja, Trigo, Hortalizas, Vegetales etc. Posee una variedad de flora en el sector: Alguirder, Alfalfa, Grama, Ortiga, El Fuma, Trébol, Aloflige, Hilapo, Amapola etc. Tiene una variedad de fauna en el sector: Pájaros, Tórtolas, Mirlos, Huirachuro, Yuto, Palomas, Colibrí, Picaflor, Gavilán, sapo, ranas, lagartijas, arañas, etc (CEGESPU (2011)) 
Cabe recalcar su territorio es amplio para fomentar el turismo y versátil en cuanto a su producción agrícola donde se pueden encontrar productos de zonas humedas y áridas además la gran variedad de fauna que existe dentro del entorno hace que sea un territorio apto para proyectar mejorar a futuro

La población desagregada por sexo y grupos de edad en la parroquia Santiago de Calpi en población desagregada por sexo y grupo de edad cuenta con una población total de 6469 habitantes, de los cuales 3457 pertenecen a la población de mujeres (53\%) y 3012 pertenece a la población masculina (47\%). El analfabetismo en mujeres se presenta en $31,67 \%$, mientras que en varones un $15,51 \%$. La Tasa de crecimiento parroquial es de 2,13 se considera que se encuentra dentro de los parámetros normales. (CEGESPU (2011))

La población desagregada por sexo y grupos de edad es el cálculo de indicadores para determinar el número de habitantes desagregando el sexo en masculino y femenino para el PDOT se desagrego en grupos donde se el total de habitantes de entre 15 a 29, 30 a 49, 50 a 64 años, esto permitió gestionar de mejor manera los recursos para los habitantes de la Parroquia Calpi.

Planificación del desarrollo parroquial

La construcción de los PDOT debe propiciar un proceso armónico y equilibrado dentro del sistema territorial, de manera que los esfuerzos entre niveles de gobierno se complementen y potencien de manera integrada (Lootugs, 2019, art. 11)

Al hablar de una planificación del desarrollo parroquial destacamos las técnicas realizadas de una manera coherente para que sus actividades y recursos sean bien invertidos que puedan brindar los beneficios a la población vulnerable, implementando estrategias de desarrollo socioeconómico, geográficas y culturales.

Al realizar el análisis de las problemáticas a través de las entrevistas con las autoridades y personas de las diferentes comunidades, mesas de concertación y observación directa se ha determinado diferentes causas y efectos para poder brindar una serie de estrategias y proponer programas y proyectos que veremos más adelante.

En el grafico 1 se podemos evidenciar las posibles causas como son carencia de organizaciones agras productivas en la parroquia, ausencia de difusión de las actividades que realiza las autoridades, baja coordinación con las Instituciones nacionales, ausencia de promotores en la Parroquia, escasa coordinación con las instituciones internacionales a lo que conlleva a los siguientes efectos ausencia de organización en la parroquias, Bajos ingresos de los productores, desconocimiento de las actividades realizadas por las autoridades, poca asistencia a las necesidades de la Parroquia, carece de recolección de indicadores, carece de ayuda la Parroquia lo que nos lleva a obtener una baja cobertura en la planificación, gestión y organización de la parroquial (PDOT, Calpi (2013)) 


\section{Grafico 1. Análisis de la problemática}

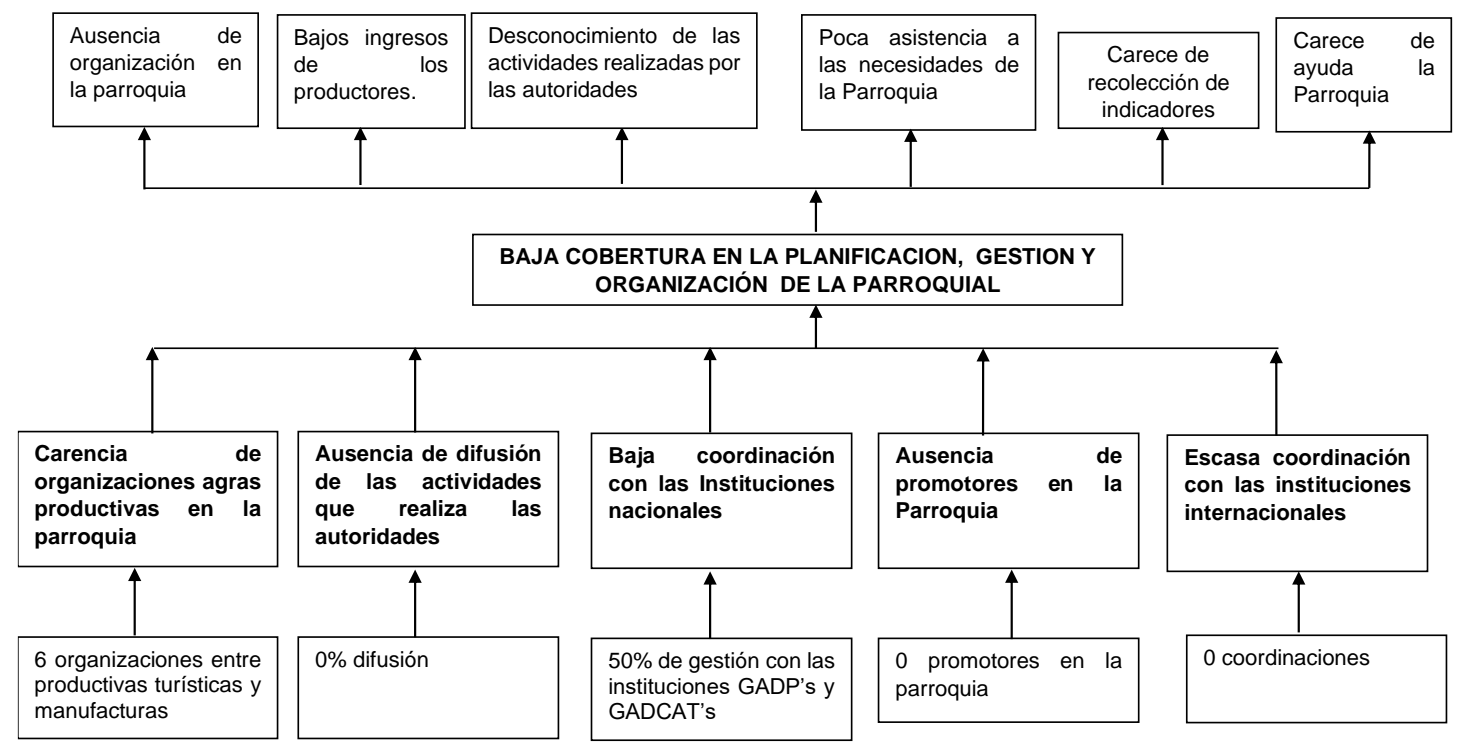

Fuente: CEGESPU 2011

Elaborado por: Consultor

Grafico 2. Análisis de los objetivos

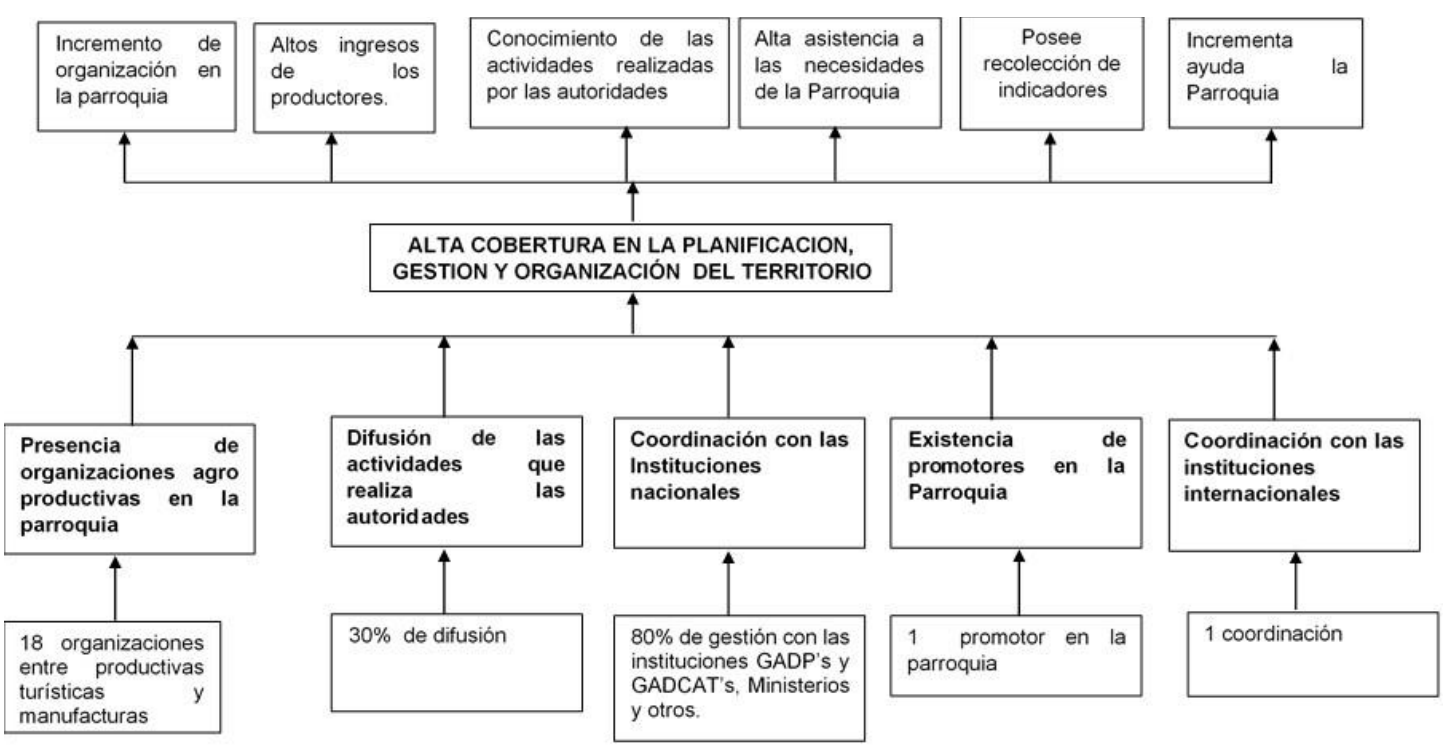

Fuente: CEGESPU 2011

Elaborado por: Consultor

La metodología de marco lógico es una herramienta que ha venido empleado la banca multilateral, organizaciones no gubernamentales para el desarrollo (ONGD) y organismos nacionales para facilitar el proceso de planificación, seguimiento y evaluación de los 
proyectos. Así, se solventan con ella algunos problemas que se venían presentando en la ejecución de los proyectos, pues ofrece una serie de ventajas sobre otros enfoques menos estructurados. (Sánchez N, (2007)

En el proceso de construcción del marco lógico con el grupo técnico determina el fin, propósitos, componentes los cuales describen los programas que intervienen en la competencia del desarrollo parroquial, mientras que los indicadores se despliega el listado de proyectos y los años en que se van a ejecutar con la participación de las personas que van a colaborar a futuro, se ubica también los medios de verificación que son las evidencias documentales del cumplimento de la ejecución de los programas y proyectos, los supuestos menciona las posibles acciones a suceder en los años establecidos estos pueden ser positivos o negativos, por último se describe las actividades donde se menciona los programas y proyectos con su respectivo presupuesto que se van a ejecutar y el número de personas a ser capacitados.

Tabla 1: Marco Lógico

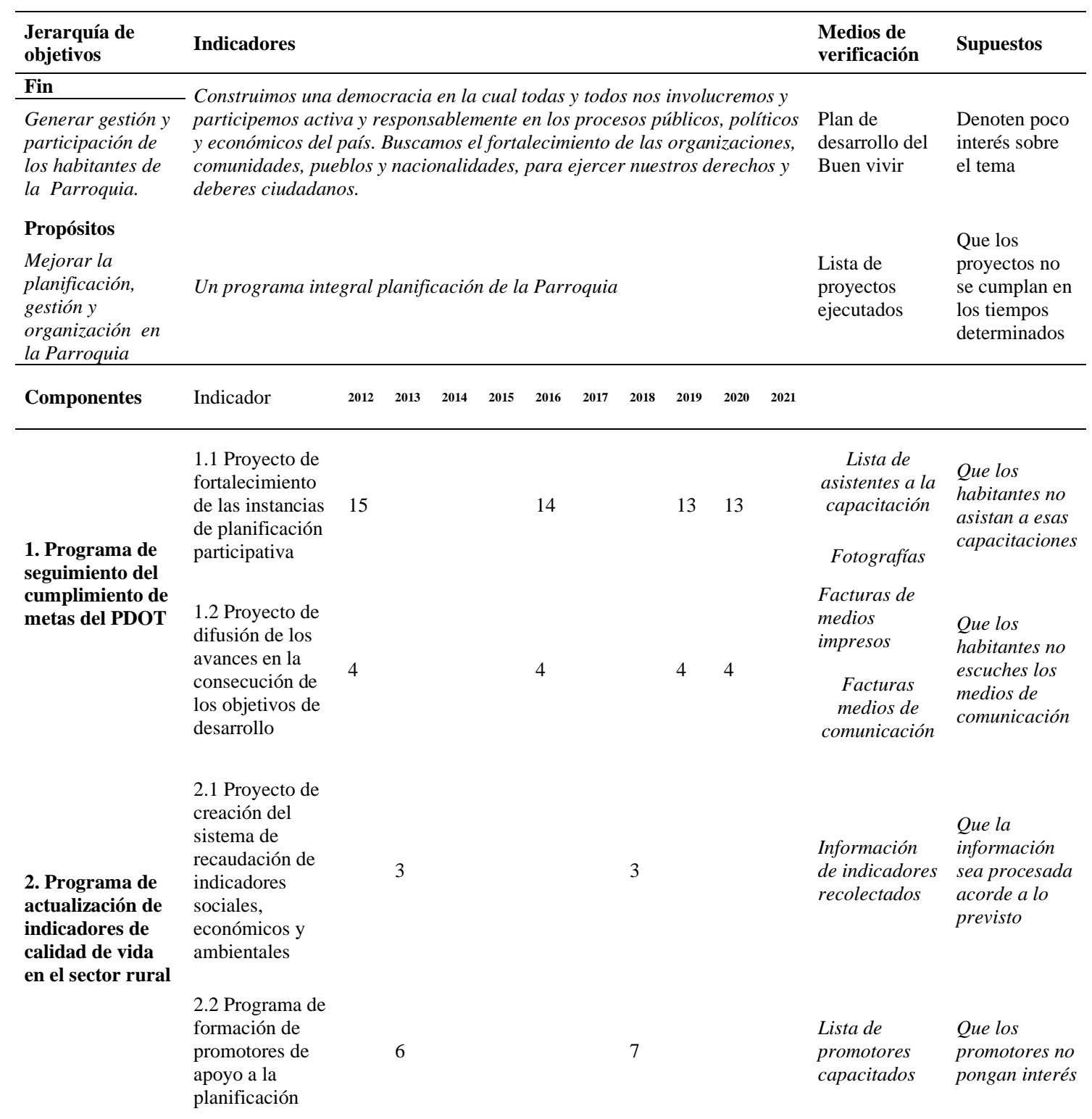


ISSN: 2600-5859

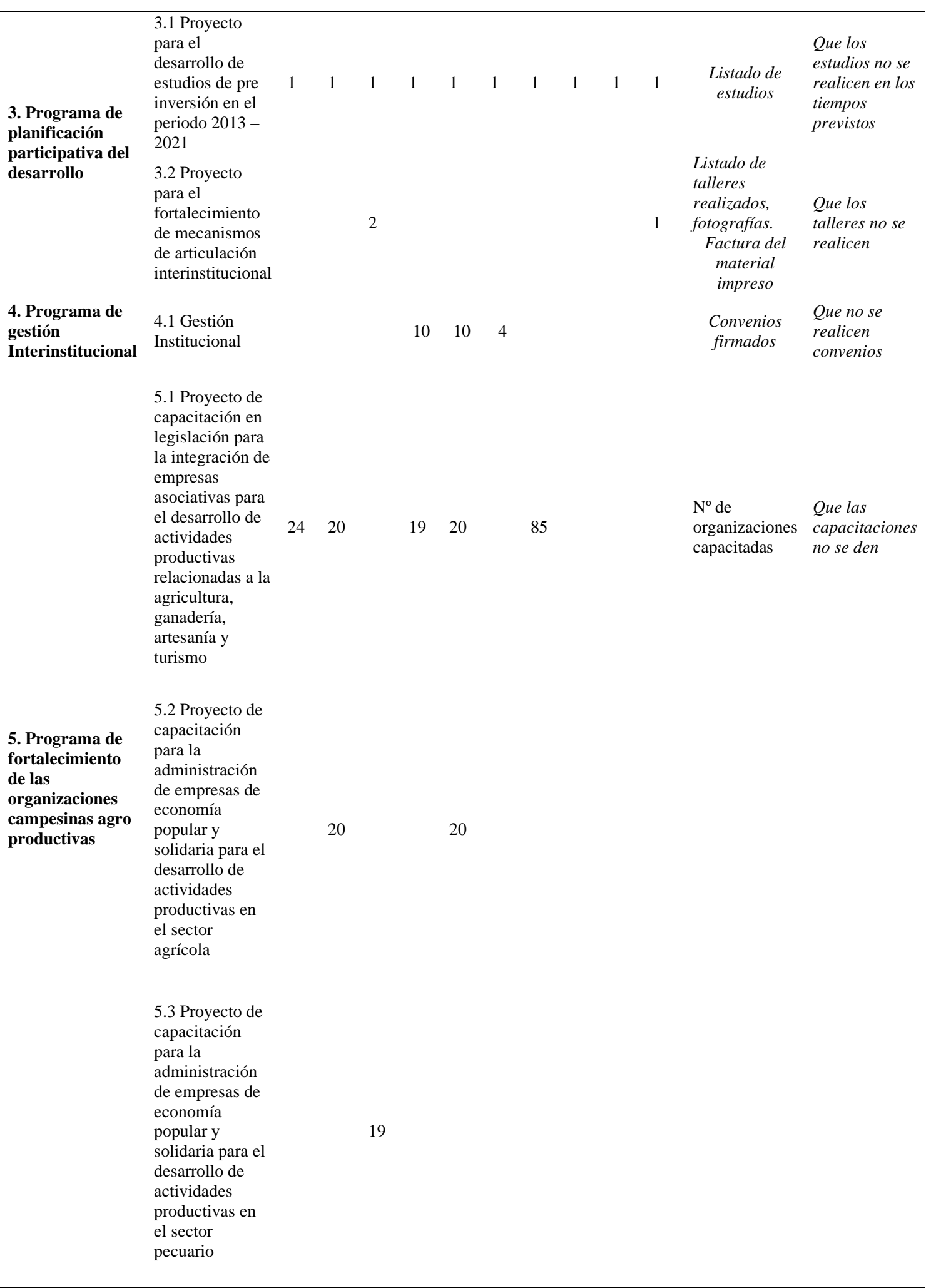


5.4 Proyecto de

capacitación

para la

administración

de empresas de

economía

popular y

solidaria para el

desarrollo de

actividades

productivas en

el sector

artesanal

5.5 Proyecto de

capacitación

para la

administración

de empresas de

economía

popular y

solidaria para el

desarrollo de

actividades

productivas en

el sector turismo

ACTIVIDADES PRESUPUESTO

1.1 Proyecto de

fortalecimiento de

las instancias de

planificación

participativa

1.2 Proyecto de difusión de los avances en la consecución de los objetivos de desarrollo

2.1 Proyecto de creación del sistema de recaudación de indicadores

sociales,

económicos y

ambientales

2.2 Programa de

formación de

promotores de

apoyo a la

planificación

3.1 Proyecto para el desarrollo de

estudios de

preinversión en el

periodo 2013 -

2021

3.2 Proyecto para

el fortalecimiento

de mecanismos de articulación

interinstitucional

4262
Lista de

asistentes a la

capacitación

Que los

Fotografias

asistan a esas

capacitaciones

Facturas de

medios

impresos

Que los

habitantes no

Facturas escuches los

medios medios de

comunicación

comunicación

Información

Que la

información

de indicadores sea procesada recolectados acorde a lo previsto

Lista de

Que los

promotores

promotores no

capacitados

pongan interés

Listado de

Que los

estudios

estudios no se realicen en los

tiempos

previstos

Listado de

talleres

Que los

realizados,

talleres no se

fotografías. 
ISSN: 2600-5859

4.1 Gestión Institucional

4380

5.1 Proyecto de

capacitación en

legislación para la

integración de

empresas

asociativas para el

desarrollo de

actividades

productivas

relacionadas a la

agricultura,

ganadería,

artesanía y

turismo

5.2 Proyecto de

capacitación para

la administración

de empresas de

economía popular

y solidaria para el

desarrollo de

actividades

productivas en el

sector agrícola

5.3 Proyecto de

capacitación para

la administración

de empresas de

economía popula

y solidaria para el

desarrollo de

actividades

productivas en el

sector pecuario

5.4 Proyecto de

capacitación para

la administración

de empresas de

economía popula

y solidaria para el

desarrollo de

actividades

productivas en el

sector artesanal
Factura del

material

impreso

Convenios

firmados

Que no se

realicen

convenios

$\mathrm{N}^{\mathrm{o}}$ de personas

Que las

capacitadas

personas no

asistan

$\begin{array}{ll}\mathrm{N}^{\mathrm{o}} \mathrm{de} & \text { lleguen a } \\ \text { organizaciones } & \text { forman } \\ \text { capacitadas } & \text { ninguna } \\ & \text { empresa }\end{array}$

$\mathrm{N}^{\mathrm{o}}$ de personas

Que las

capacitadas personas no asistan

Fuente: CEGESPU 2011

Elaborado por: Consultor 
Objetivos de desarrollo de planificación territorial

Según la CEPAL 2019 la Agenda 2030 para el desarrollo sostenible y los objetivos de desarrollo sostenible (ODS) proponen una ruta para acometer los grandes desafíos del desarrollo, que solo podrá hacerse realidad a través del diseño, implementación, seguimiento y evaluación de políticas públicas que integren simultáneamente las dimensiones económica, social y ambiental.

Los objetivos de desarrollo de GAD parroquial Calpi fueron realizados en base a la competencia del desarrollo parroquial que describe la participación de los habitantes de la parroquia, convenios con otras instituciones, además cuenta un escenario deseado el cual es el optimista según los porcentajes enunciados y el escenario factible o meta mínima que es donde se desglosa los porcentajes según la meta deseada.

Tabla 2: Objetivo de desarrollo

Objetivo de desarrollo $\quad$ Escenario deseado $\quad \begin{aligned} & \text { Escenario } \\ & \text { mínima) }\end{aligned} \quad$ factible(Meta

\begin{tabular}{|c|c|c|}
\hline $\begin{array}{lcr}\text { Generar } & \text { gestión } & \text { y } \\
\text { participación } & \text { de } & \text { los } \\
\text { habitantes de la parroquia }\end{array}$ & $\begin{array}{l}\text { El } 100 \% \text { de la población se } \\
\text { involucre en forma activa y } \\
\text { responsable en la } \\
\text { planificación de obras }\end{array}$ & $\begin{array}{l}30 \% \text { de los ciudadanos acepten } \\
\text { la gestión y se integren a } \\
\text { mejorar el desarrollo de la } \\
\text { parroquia junto al Gobierno } \\
\text { parroquial. }\end{array}$ \\
\hline $\begin{array}{l}\text { Planificar acorde a otras } \\
\text { instituciones para obtener } \\
\text { mejores beneficios para los } \\
\text { ciudadanos }\end{array}$ & $\begin{array}{l}\text { Que la planificación sea en } \\
\text { beneficio del } 80 \% \text { de la } \\
\text { población llegando a los } \\
\text { sectores más necesitados las } \\
\text { obras }\end{array}$ & $\begin{array}{l}\text { Llegar al } 30 \% \text { con mejor } \\
\text { planificación de obras }\end{array}$ \\
\hline $\begin{array}{lr}\begin{array}{l}\text { Gestionar } \\
\text { instituciones }\end{array} & \begin{array}{r}\text { otras } \\
\text { obras }\end{array} \\
\text { prioritarias para la parroquia }\end{array}$ & $\begin{array}{l}\text { Tratar de llegar al } 80 \% \text { de las } \\
\text { instituciones para realizar } \\
\text { obras conjuntas }\end{array}$ & $\begin{array}{l}\text { Que el } 30 \% \text { de las instituciones } \\
\text { de índole superior fomenten } \\
\text { obras junto al gobierno } \\
\text { parroquial }\end{array}$ \\
\hline
\end{tabular}

Fuente: CEGESPU 2011

Elaborado por: Consultor

Modalidad de gestión por competencias

La modalidad de gestión por competencias se determina los proyectos a implementarse en conjunto con el equipo técnico y las autoridades en base a la planificación del desarrollo parroquial y su modalidad de gestión esta describe si esta se desarrollara como gestión compartida, Gestión directa, cogestión comunitaria, al hablar de gestión compartida sería el GAD parroquial en conjunto con el GAD municipal, GAD provincial en lo que se refiere gestión directa que se refiere al Gobierno autónomo descentralizado de Calpi será el encargado de desarrollar y cogestión comunitaria el cual desarrollara en conjunto el GAD parroquial y las comunidades que sean beneficiadas(mingas) o con el apoyo de Empresas de economía popular solidaria, Empresas públicas. 
Tabla 3: Proyectos previstos a Implementarse

\section{PROYECTOS \\ IMPLEMENTARSE \\ PREVISTOS}

A. PLANIFICACION DEL

DESARROLLO PARROQUIAL

1. Programa de seguimiento del cumplimiento de metas del PDOT

2. Programa de actualización de indicadores de calidad de vida en el sector rural

3. Programa de planificación participativa del desarrollo

Gestión directa

- Cogestión comunitaria.

4. Programa de gestión Interinstitucional - Gestión compartida

- Gestión directa

Fuente: CEGESPU 2011

Elaborado por: Consultor

Plan plurianual de acciones metas mínimas

El plan plurianual de acciones de metas mínimas comprende los programas, proyectos indicadores y se describe el horizonte de planificación del año 2012 al 2021 donde da a conocer las descripción de lo que contiene cada indicador por proyecto o programa

TABLA 4: Plan Plurianual de acciones metas mínimas

\begin{tabular}{|c|c|c|c|c|c|c|c|c|c|c|c|c|c|}
\hline \multirow{2}{*}{ Programa } & & \multicolumn{12}{|c|}{ Horizonte de planificación $(*)$} \\
\hline & & \multicolumn{2}{|l|}{ INDICADOR } & 2012 & $\begin{array}{l}20 \\
13\end{array}$ & $\begin{array}{l}20 \\
14\end{array}$ & $\begin{array}{l}20 \\
15\end{array}$ & $\begin{array}{l}20 \\
16\end{array}$ & $\begin{array}{l}20 \\
17\end{array}$ & $\begin{array}{l}20 \\
18\end{array}$ & $\begin{array}{l}20 \\
19\end{array}$ & $\begin{array}{l}20 \\
20\end{array}$ & $\begin{array}{l}20 \\
21\end{array}$ \\
\hline $\begin{array}{c}1 . \\
\text { Programa } \\
\text { de } \\
\text { seguimient }\end{array}$ & $\begin{array}{l}\text { Proyecto de fortalecimiento de las } \\
\text { instancias de planificación } \\
\text { participativa }\end{array}$ & $\begin{array}{l}\mathrm{N}^{\circ} \text { de miembros de las } \\
\text { instancias de participación } \\
\text { capacitados }\end{array}$ & 15 & & & & & 14 & & & 13 & 13 & \\
\hline $\begin{array}{l}\text { o del } \\
\text { cumplimie } \\
\text { nto de }\end{array}$ & $\begin{array}{l}\text { Proyecto de difusión de los } \\
\text { avances en la consecución de los }\end{array}$ & $\begin{array}{l}N^{0} \text { de unidades de material } \\
\text { trípticos }\end{array}$ & 228 & & & & & $\begin{array}{c}26 \\
3\end{array}$ & & & $\begin{array}{c}28 \\
6\end{array}$ & $\begin{array}{c}29 \\
4\end{array}$ & \\
\hline $\begin{array}{l}\text { metas del } \\
\text { PDOT }\end{array}$ & objetivos de desarrollo & $\begin{array}{l}N^{o} \text { de medios de } \\
\text { comunicación radiales } \\
\text { contratados }\end{array}$ & 3 & & & & & 2 & & & 2 & 2 & \\
\hline $\begin{array}{c}2 . \\
\text { Programa }\end{array}$ & & $\begin{array}{l}N^{o} \text { de indicadores sociales } \\
\text { levantados por año }\end{array}$ & & & 1 & & & & & 1 & & & \\
\hline $\begin{array}{c}\text { de } \\
\text { actualizaci } \\
\text { ón de } \\
\text { indicadore }\end{array}$ & $\begin{array}{l}\text { Proyecto de creación del sistema } \\
\text { de recaudación de indicadores } \\
\text { sociales, económicos y ambientales }\end{array}$ & $\begin{array}{l}\mathrm{N}^{\circ} \text { de indicadores } \\
\text { económicos levantados por } \\
\text { año }\end{array}$ & & & 1 & & & & & 1 & & & \\
\hline $\begin{array}{l}\text { s de } \\
\text { calidad de }\end{array}$ & & $\begin{array}{l}N^{\circ} \text { de indicadores sociales } \\
\text { levantados por año }\end{array}$ & & & 1 & & & & & 1 & & & \\
\hline $\begin{array}{l}\text { vida en el } \\
\text { sector } \\
\text { rural }\end{array}$ & $\begin{array}{c}\text { Programa de formación de } \\
\text { promotores de apoyo a la } \\
\text { planificación }\end{array}$ & $\begin{array}{l}N^{\circ} \text { de promotores de apoyo } \\
\text { a la planificación formados }\end{array}$ & & & 6 & & & & & 7 & & & \\
\hline $\begin{array}{c}3 . \\
\text { Programa } \\
\text { de } \\
\text { planificaci } \\
\text { on } \\
\text { participati }\end{array}$ & $\begin{array}{c}\text { Proyecto para el desarrollo de } \\
\text { estudios de preinversión en el } \\
\text { periodo } 2013 \text { - } 2021\end{array}$ & $\begin{array}{l}\mathbf{N}^{0} \text { de estudios de } \\
\text { preinversión en materia } \\
\text { productiva elaborados } \\
\mathbf{N}^{0} \text { de estudios de } \\
\text { preinversión en materia } \\
\text { ambiental elaborados }\end{array}$ & 1 & & & 1 & 1 & 1 & & 1 & 1 & 1 & \\
\hline
\end{tabular}


ISSN: 2600-5859

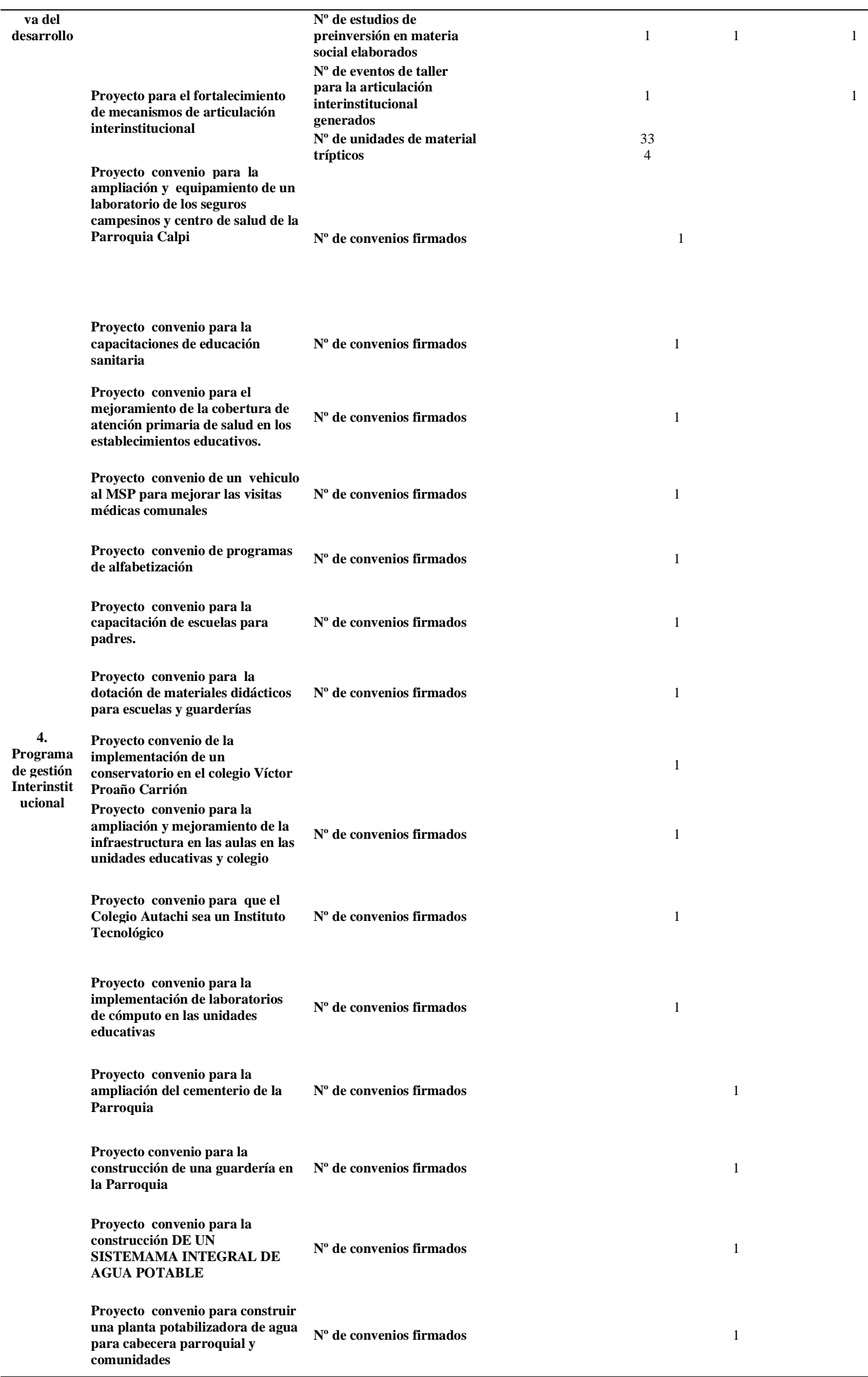


Proyecto convenio para la elaboración de un programa urbanístico de la cabecera Parroquia y comunidades

Proyecto convenio para el desarrollo integral de alcantarillado para la

Proyecto convenio para la construcción de un sistema de riego (a comunidades y barrios)

$\mathbf{N}^{\circ}$ de convenios firmados

Proyecto convenio con

cooperativas de transporte

urbano, rural para mejoramiento

$\mathrm{N}^{\circ}$ de convenios firmados

1

del servicio y horarios

Proyecto convenio asfaltado de vías de tierra

Proyecto convenio de la construcción de un sistema integral de agua de riego

Proyecto convenio EMMPA

empresas privadas para la entrega $\mathbf{N}^{\circ}$ de convenios firmados de productos

Proyecto convenios con el

MAGAP, INIAP, FAO,

ministerios y otras instituciones

afines para asesoramiento técnico

$\mathrm{N}^{\mathrm{o}}$ de convenios firmados en la Parroquia

Convenio para la elaboración de proyectos ambientales y sociales con las empresas que habitan en el $\mathbf{N}^{\circ}$ de convenios firmados territorio de la Parroquia 1 para la dotación de personal
policial, patrullas, motos para la
vigilancia de la Parroquia.

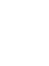


TABLA 5: Inversión asignada por presupuestación participativa en las instancias de Participación Ciudadana.

\begin{tabular}{|c|c|c|c|c|c|c|c|c|c|c|c|c|c|}
\hline \multirow{2}{*}{ AÑO } & \multirow{2}{*}{2012} & \multirow{2}{*}{2013} & \multirow{2}{*}{2014} & \multirow{2}{*}{2015} & \multirow{2}{*}{2016} & \multirow{2}{*}{2017} & \multirow{2}{*}{2018} & \multirow{2}{*}{2019} & \multirow{2}{*}{2020} & \multirow{2}{*}{2021} & $\begin{array}{l}\% \quad \text { asignados por } \\
\text { competencias } 2013\end{array}$ & \multirow{2}{*}{$\begin{array}{l}\% \quad \text { asignados } \\
\text { competencias } \\
\text { años }\end{array}$} & \multirow{2}{*}{$\begin{array}{l}\text { por } \\
\text { demas }\end{array}$} \\
\hline & & & & & & & & & & & Asamblea & & \\
\hline PRESUPUESTO TOTAL & $\begin{array}{r}\$ \\
160.77 \\
2,74\end{array}$ & $\begin{array}{r}\$ \\
196.59 \\
7,53\end{array}$ & $\begin{array}{r}\$ \\
202.49 \\
5,46\end{array}$ & $\begin{array}{r}\$ \\
208.57 \\
0,32\end{array}$ & $\begin{array}{r}\$ \\
214.82 \\
7,43\end{array}$ & $\begin{array}{r}\$ \\
221.27 \\
2,25\end{array}$ & $\begin{array}{r}\$ \$ \\
227.91 \\
0,42\end{array}$ & $\begin{array}{r}\$ \\
234.74 \\
7,73\end{array}$ & $\begin{array}{r}\$ \\
241.79 \\
0,16\end{array}$ & $\begin{array}{r}\$ \\
249.04 \\
3,87\end{array}$ & & & \\
\hline GASTO CORRIENTE $(30 \%)$ & $\begin{array}{r}\$ \\
48.231 \\
, 82 \\
\$\end{array}$ & $\begin{array}{r}\$ \\
58.979 \\
, 26 \\
\$\end{array}$ & $\begin{array}{r}\$ \\
60.748 \\
, 64 \\
\$\end{array}$ & $\begin{array}{r}\$ \\
62.571 \\
, 10 \\
\$\end{array}$ & $\begin{array}{r}\$ \\
64.448 \\
, 23 \\
\$\end{array}$ & $\begin{array}{r}\$ \\
66.381 \\
, 68 \\
\$\end{array}$ & $\begin{array}{r}\$ \\
68.373 \\
, 13 \\
\$\end{array}$ & $\begin{array}{r}\$ \\
70.424 \\
.32 \\
\$\end{array}$ & $\begin{array}{r}\$ \\
72.537 \\
, 05 \\
\$\end{array}$ & $\begin{array}{r}\$ \\
74.713 \\
, 16 \\
\$\end{array}$ & $30 \%$ & & $30 \%$ \\
\hline PRESUPUESTO DE INVERSIÓN (70\%) & $\begin{array}{r}112.54 \\
0,91\end{array}$ & $\begin{array}{r}137.61 \\
8,27\end{array}$ & $\begin{array}{r}141.74 \\
6,82\end{array}$ & $\begin{array}{r}145.99 \\
9,22\end{array}$ & $\begin{array}{r}150.37 \\
9,20\end{array}$ & $\begin{array}{r}154.89 \\
0,58\end{array}$ & $\begin{array}{r}159.53 \\
7,29\end{array}$ & $\begin{array}{r}164.32 \\
3,41\end{array}$ & $\begin{array}{r}169.25 \\
3,11\end{array}$ & $\begin{array}{r}174.33 \\
0,71\end{array}$ & $70 \%$ & & $70 \%$ \\
\hline $\begin{array}{lll}\text { PRESUPUESTO } & \text { GRUPOS } & \text { DE } \\
\text { ATENCIÓN PRIORITARIA } & (10 \% & \text { DE } \\
\text { INVERSIÓN) } & & \end{array}$ & $\begin{array}{r}\$ \\
11.254 \\
, 09\end{array}$ & $\begin{array}{r}\$ \\
19.659 \\
75\end{array}$ & $\begin{array}{r}\$ \\
20.249 \\
, 55\end{array}$ & $\begin{array}{r}\$ \\
20.857 \\
, 03\end{array}$ & $\begin{array}{r}\$ \\
21.482 \\
, 74\end{array}$ & $\begin{array}{r}\$ \\
22.127 \\
, 23\end{array}$ & $\begin{array}{r}\$ \\
22.791 \\
, 04\end{array}$ & $\begin{array}{r}\$ \\
23.474 \\
, 77\end{array}$ & $\begin{array}{r}\$ \\
24.179 \\
, 02\end{array}$ & $\begin{array}{r}\$ \\
24.904 \\
, 39\end{array}$ & $10 \%$ & & $10 \%$ \\
\hline $\begin{array}{l}\text { PRESUPUESTO DE INVERSIÓN PARA } \\
\text { EL EJERCICIO DE COMPETENCIAS } \\
\text { EXCLUSIVAS }\end{array}$ & $\begin{array}{r}\$ \\
101.28 \\
6,82\end{array}$ & $\begin{array}{r}\$ \\
117.95 \\
8,52\end{array}$ & $\begin{array}{r}\$ \\
121.49 \\
7,27\end{array}$ & $\begin{array}{r}\$ \\
125.14 \\
2,19\end{array}$ & $\begin{array}{r}\$ \\
128.89 \\
6,46\end{array}$ & $\begin{array}{r}\$ \\
132.76 \\
3,35\end{array}$ & $\begin{array}{r}\$ \\
136.74 \\
6,25\end{array}$ & $\begin{array}{r}\$ \\
140.84 \\
8,64\end{array}$ & $\begin{array}{r}\$ \\
145.07 \\
4,10\end{array}$ & $\begin{array}{r}\$ \\
149.42 \\
6,32\end{array}$ & & & \\
\hline $\begin{array}{ll}\text { A. } \quad \text { PLANIFICACIÓN } & \text { DEL } \\
\text { DESARROLLO PARROQUIAL } & \end{array}$ & $\begin{array}{r}\$ \\
5.064, \\
34\end{array}$ & $\begin{array}{r}\$ \\
11.795 \\
, 85\end{array}$ & $\begin{array}{r}\$ \\
6.074, \\
86\end{array}$ & $\begin{array}{r}\$ \\
6.257 \\
11\end{array}$ & $\begin{array}{r}\$ \\
6.444, \\
82\end{array}$ & $\begin{array}{r}\$ \\
6.638, \\
17\end{array}$ & $\begin{array}{r}\$ \\
6.837 \\
31\end{array}$ & $\begin{array}{r}\$ \\
7.042, \\
43\end{array}$ & $\begin{array}{r}\$ \\
7.253, \\
70\end{array}$ & $\begin{array}{r}\$ \\
7.471, \\
32\end{array}$ & $10 \%$ & & $5 \%$ \\
\hline
\end{tabular}

Fuente: CEGESPU 2011

Elaborado por: Consultor

\section{PLAN PLURIANUAL DE EJECUCIÓN PRESUPUESTARIA}

El Plan Plurianual de Inversión Pública (PPIP) comprende los programas y proyectos programados por las entidades del Estado para su ejecución y cuyo financiamiento está incluido en el Presupuesto General del Estado (PGE). Estas inversiones se encuentran alineadas con los objetivos de la planificación y son consistentes con los planes anuales. De esta forma, el PPIP se convierte en una herramienta de gestión pública necesaria para la eficiente implementación de un sistema presupuestario por resultados (Gómez y Martínez, 2008).

De esta manera enfocamos los recursos en la competencia analizada que corresponde a la planificación del desarrollo parroquial el mismo que desglosa los programas y su correspondiente distribución del presupuesto en los diferentes años para su correcta ejecución.

TABLA 8: PLAN PLURIANUAL DE EJECUCIÓN PRESUPUESTARIA

\begin{tabular}{lllllllllllll}
\hline \multirow{2}{*}{ Competencias } & Programas a ejecutarse & \multicolumn{1}{c}{ Horizonte de planificación (*) } \\
\cline { 3 - 10 } & & 2012 & 2013 & 2014 & 2015 & 2016 & 2017 & 2018 & 2019 & 2020 & 2021 \\
\hline
\end{tabular}

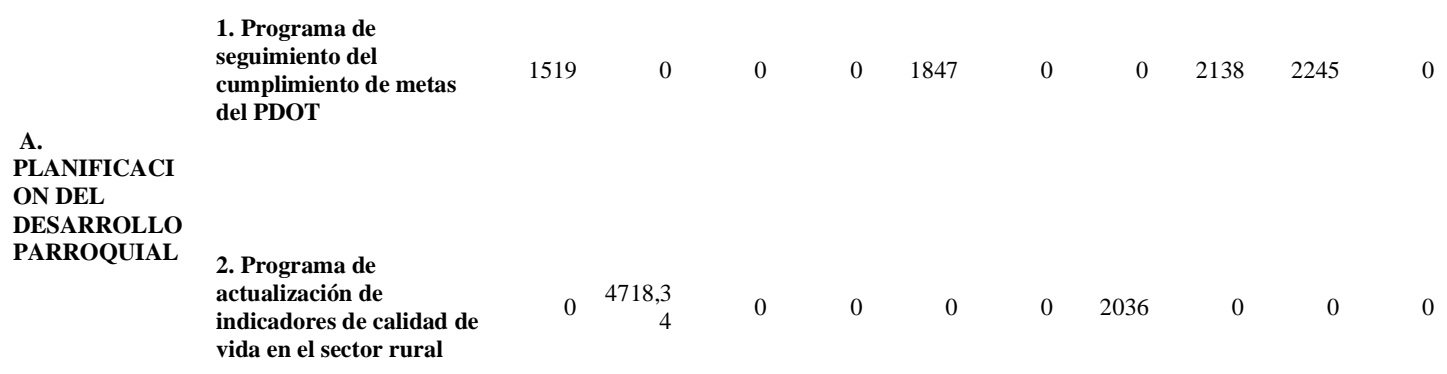




\begin{tabular}{|c|c|c|c|c|c|c|c|c|c|c|}
\hline $\begin{array}{l}\text { 3. Programa de } \\
\text { planificación }\end{array}$ & 3545,0 & 2359,1 & 5583,4 & 4103,8 & 4309,0 & 4524,4 & 4072,0 & 4988,2 & 5237,6 & 7856,4 \\
\hline $\begin{array}{l}\text { participativa del } \\
\text { desarrollo }\end{array}$ & 4 & 7 & 4 & 3 & 2 & 7 & 2 & 3 & 4 & 6 \\
\hline $\begin{array}{l}\text { 4. Programa de gestión } \\
\text { Interinstitucional }\end{array}$ & 0 & 0 & 0 & $\begin{array}{r}1758,7 \\
8\end{array}$ & 0 & $\begin{array}{r}1939,0 \\
6\end{array}$ & 509 & 0 & 0 & 0 \\
\hline 5.Gestion de Riesgos & & $\begin{array}{r}1179,5 \\
9\end{array}$ & & & & & & & & \\
\hline 6, Estudios técnicos & & $\begin{array}{r}3538,7 \\
6 \\
\end{array}$ & & & & & & & & \\
\hline
\end{tabular}

Fuente: CEGESPU 2011

Elaborado por: Consultor

\section{Conclusiones}

- Planificación del desarrollo parroquial a través del PDOT se expresa como una herramienta importante para la gestión de la parroquia cabe recalcar que es de suma importancia mantener una armonía territorial con sus habitantes, para poder generar un trabajo conjunto entre las partes, además en importante trabajar mancomunadamente entre GADS Cantonales y GADS provinciales para poder cumplir con todos los objetivos estratégicos para el bienestar de la Parroquia y sus comunidades

- Mantener actualizado los indicadores de calidad de vida en el sector de las comunidades esto permitirá actualizar el presupuesto y dirigir sus esfuerzos para los sectores menos beneficiados, lo cual podrá mejor el estilo de vida de las personas que tengas los diferentes beneficios sean estos proyectos $u$ obras de servicios básicos

- Implementar las capacitaciones a los personeros de las comunidades para generar conocimientos en participación ciudadana y control social para que puedan tomar acciones positivas en cada una de los proyectos y programas a realizarse

- Generar convenios interinstitucionales de la parroquia para poder mejorar la parte de salubridad, educación, servicios básicos, mejoramiento urbanístico, alcantarillado comunitario, mejoramiento en el transporte, mejoramiento vial, sistema de riego para comunidades y parroquia, crear microempresa en la parroquia, mitigación ambiental de la parroquia y sus comunidades

- El presente trabajo desarrollado será una herramienta en la competencia planificación del territorio lo cual es muy importante ya que se deberá organizar y empatar con las demás competencias donde recaiga en los programas y proyectos los mismos que permitirán

\section{Bibliografía}

Alberich, T., \& Sotomayor, E. (2014). Planificacíon, Gestión y Evaluación Manual básico para la Acción Social. Madrid: DYKINSON, S.L. Obtenido de https://books.google.com.ec/books?id=C5_bBQAAQBAJ\&pg=PA107\&dq=plan ificaci\%C3\%B3n+social++++2015\&hl=es\&sa=X\&ved=0ahUKEwjBgcaZuJfcA 
hWpwFkKHUD7A7EQ6AEIJjAA\#v=onepage \&q=planificaci\%C3\%B3n\%20so cial $\% 20 \% 20 \% 20 \% 202015 \& \mathrm{f}=$ false

Asamblea Constitucional de Montecristi . (2008). Constitución de la República del Ecuador. Quito: Ediciones Legales.

CEGESPU, (2011). Plan de Desarrollo y Ordenamiento Territorial Parroquia Calpi: Gobierno autónomo descentralizado Calpi.

Guzmán Saavedra , R., Castro Zea, L. E., Restrepo Quintero, O., \& Rojas Rojas, A. (2001). Planificación del Desarrollo . Bogota: Editora Géminis Ltda.

Serrano, G. P. (2016). Diseño de Proyectos Sociales. Madrid: Narcea, S.A de Ediciones. Obtenido de https://books.google.com.ec/books?id=JH29DAAAQBAJ\&printsec=frontcover $\& d q=$ lanificaci $\%$ C3\%B3n+social++++2016\&hl=es\&sa=X\&ved=0ahUKEwigp $5 \mathrm{i} 2 \mathrm{xpfcAhUNr} 1 \mathrm{kKHSEfA0AQ6AEIMzAC \# v=onepage} \& \mathrm{q}=$ planificaci\%C3\%B $3 \mathrm{n} \% 20$ social $\% 20 \% 20 \% 20 \% 202016 \& \mathrm{f}=$ false

Fernández, S. P. 2002. Investigación cuantitativa y cualitativa. Cad Aten primaria complejo Hospitalario Juan Canalejo. Coruña, España. 76-78 p.

https://www.planificacion.gob.ec/wp-content/uploads/downloads/2019/08/Folletosautoridades-parroquiales.pdf

Lootugs: Ley Orgánica de Ordenamiento Territorial, Uso y Gestión del Suelo (2018). Quito, Ecuador: Registro Oficial Suplemento No. 790, de 5 de julio de 2016. Última modificación: 23 de octubre de 2018.

Sánchez N, (2007) El marco lógico metodología para la planificación, seguimiento y evaluación de proyectos, Venezuela: revista visión general, obtenido de https://www.redalyc.org/pdf/4655/465545876012.pdf

CEPAL, (2019) Planificación para el desarrollo territorial sostenible en América Latina y el Caribe, obtenido https://repositorio.cepal.org/bitstream/handle/11362/44731/1/S1900439_es.pdf

Gómez, Juan. Martínez-Vázquez, Jorge (2008). La Programación Presupuestaria Plurianual con referencia a su práctica en el Reino Unido y Dinamarca. Georgia State University, USA. Instituto de Estudios Fiscales.

MDUV, Ministerio de Desarrollo Urbano y Vivienda (2018) Ley Orgánica de Ordenamiento Territorial, uso y Gestión de suelo Conceptos básicos, obtenido en: https://www.habitatyvivienda.gob.ec/wpcontent/uploads/downloads/2018/06/LOOTUGS-ConceptosBasicos_oficial_8M.pdf 
STPE, Secretaria Técnica de Planifica Ecuador (2019) Guía para la formulación/Actualización del Plan de Desarrollo y Ordenamiento Territorial (PDOT) Parroquial obtenido: enhttps://www.planificacion.gob.ec/wpcontent/uploads/downloads/2019/08/GUIA-PARROQUIAL-FINAL.pdf

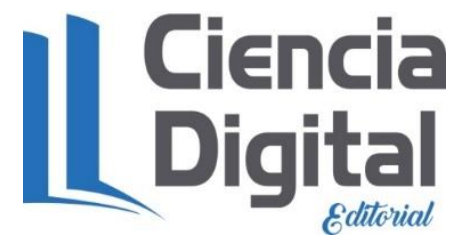




\section{PARA CITAR EL ARTÍCULO INDEXADO.}

Centeno Parra, E. X., Alarcón Parra, G. J., Pilaguano Mendoza, J. G., \& Viscarra Armijos, C. P. (2021). Elaboración del Plan de Desarrollo y Ordenamiento Territorial Caso Gobierno autónomo descentralizado de Calpi 2011 (Competencia del desarrollo parroquial). ConcienciaDigital, $4(2.2)$ $21-40$. https://doi.org/10.33262/concienciadigital.v4i2.2.1735

\section{Ciencia \\ LDigital}

El artículo que se publica es de exclusiva responsabilidad de los autores y no necesariamente reflejan el pensamiento de la Revista Conciencia Digital.

El artículo queda en propiedad de la revista y, por tanto, su publicación parcial y/o total en otro medio tiene que ser autorizado por el director de la Revista Conciencia Digital.

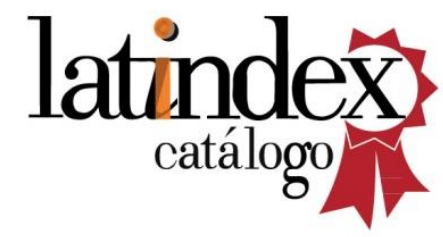

